\title{
Development of large area polycrystalline diamond detectors for fast timing application of high-energy heavy-ion beams
}

F. Schirru ${ }^{1}$, B. S. Nara Singh ${ }^{2}$, L. Scruton ${ }^{2}$, M. A. Bentley ${ }^{2}$, S. P. Fox ${ }^{2}$, A. Lohstroh ${ }_{2}^{1}$ P. J. Sellin $^{1}$, *A. Banu ${ }^{3}$, M. McCleskey ${ }^{3}$, B. T. Roeder ${ }^{3}$, E. Simmons ${ }^{3}$, A. A. Alharbi ${ }^{3}$, L. Trache ${ }^{3}$, M. Freer $^{4}$, and D. Parker ${ }^{4}$

${ }^{I}$ Department of Physics, University of Surrey, Guildford, GU2 7XH, United Kingdom

${ }^{2}$ Department of Physics, University of York, Heslington, York YO10 5DD, United Kingdom

${ }^{3}$ Cyclotron Institute, Texas A \& M University, College Station, Texas, 77843, USA

${ }^{4}$ School of Physics and Astronomy, University of Birmingham, Edgbaston, Birmingham, B15

2TT, United Kingdom

\begin{abstract}
We have studied the effects of electrode fabrication and detector capacitance on the time resolution of large area electronic grade polycrystalline diamond sensors, made using chemical vapour deposition, that are suitable for time of flight measurements of heavy ions at relativistic velocities. Sensors were prepared both in house, with Al or Au metal contacts, and commercially fabricated with $\mathrm{Au} /$ diamond-like carbon contacts. ${ }^{3} \mathrm{He},{ }^{40} \mathrm{Ar}$ and a mixture of ${ }^{20} \mathrm{Ne}$ and ${ }^{16} \mathrm{O}$ beams at $16.3,33.5$ and $21-23 \mathrm{MeV} / \mathrm{u}$, respectively were used on these devices whilst arranged in transmission geometry. Signal processing only began over one meter away from the sensors. The present approach, where we have large-area/large capacitance multi-strip detectors with processing electronics at some distance from the target, is compatible with anticipated space limitations in particle-identification and tracking setups at existing and planned nuclear fragmentation facilities. In a systematic study under these conditions, we demonstrate that the time resolution is limited by detector capacitance and energy deposition in the sensors. An intrinsic time resolution $\sigma_{\mathrm{t}}=(44 \pm 5) \mathrm{ps}$ was achieved for a diamond detector of $\sim 14 \mathrm{pF}$ capacitance. We conclude that, once further refinements are made, a large area time of flight detection system using polycrystalline diamond detectors would be able to provide time resolutions better than $40 \mathrm{ps,}$ approaching the requirement for particle-identification in relativistic fragmentation experiments, such as those at the facility for antiproton and ion research, FAIR.
\end{abstract}

\section{Introduction}

The development of radioactive beam facilities that use relativistic beams to produce exotic nuclei is presenting significant new opportunities for spectroscopy of nuclei at the very limits of existence. Facilities, such as the Facility for Antiproton and Ion Research (FAIR) [1] in Germany and Facility for Rare Isotope Beams (FRIB) [2] in Michigan USA, are in development now, and significant investments in new detector technology are required to both track and identify the exotic nuclei created, and perform the spectroscopic measurements. One requirement that is

\footnotetext{
* James Madison University, Department of Physics and Astronomy, Harrisonburg, VA 22807, USA
} 
becoming increasingly important for fragmentation facilities is the need for fast-timing detectors of large area, with timing resolution $\sigma_{t}$ of better than $40 \mathrm{ps}$ for relativistic heavy ions.

The work described here is motivated by the development of a FAIR device, namely the Lund-York-Cologne Calorimeter (LYCCA) [3-5], which is currently under development and testing. The Superconducting Fragment Separator (Super FRS) [6] at FAIR will separate beams of rare exotic nuclei, which will be focussed onto a reaction target for in-beam gamma-ray spectroscopy. LYCCA is downstream of the reaction target and is designed to track and identify the resulting nuclei - which involves using high-precision time-of-flight measurement, The current paper is not intended to describe LYCCA or any of its detectors, and for this we refer to a forthcoming paper [5] and the technical design report [3]. It is the high-precision time-of-flight requirement that motivates the current work, as diamond is one possible detector solution. Other fast-timing possibilities exist, such as fast-plastic scintillator membranes (see ref. [7]) which are used in the current version of LYCCA [7].

In a typical experiment at aforementioned facilities, timing detectors will receive a highintegrated particle flux. Therefore, radiation hardness and robustness will be a general requirement. In addition, three important issues particularly relevant to LYCCA are addressed in this paper: (i) large area coverage - a detector module of active area around $36 \mathrm{~cm}^{2}(\sim 6 \mathrm{~cm} \mathrm{X}$ $6 \mathrm{~cm}$ ) is required, due to the size of the beam spot; (ii) signal processing at a large distance - the electronics setup for processing fast-timing signals is unlikely to be placed in close proximity to the detector, due to space constraints and the expected large number of channels required and (iii) specific requirement on time resolution - simulations specifically developed for this project [4] show that in order to identify the produced nuclei, the timing detectors should have intrinsic detector timing resolutions, $\sigma_{\mathrm{t}}$, below $40 \mathrm{ps}$ when arranged in a transmission geometry with a time of flight path of only a few meters [4].

The present work investigates the potential of large area polycrystalline diamond detectors for this purpose, and in general, is of interest for any device used for tracking and identification of relativistic fragments.

There has been a large amount of developmental research work during the last few decades focussing on polycrystalline chemical vapour deposited (pcCVD) synthetic diamond material [821], which are commercially available in samples of large area. Due to the high electron and hole saturation velocity of $10^{7} \mathrm{~cm} / \mathrm{s}$, the material has been proven to provide a very good timing resolution (e.g. see refs. [9,10]). Moreover, it presents an extreme resilience to harsh environments, very high breakdown field strength $\left(10^{7} \mathrm{~V} / \mathrm{cm}\right)[11]$ and the highest room temperature thermal conductivity of any material. Large area pcCVD diamonds were chosen for this developmental study because of these suitable characteristics.

One issue of considerable importance here is the incomplete charge collection observed for pcCVD diamond caused by grain boundaries [13]. Charge collection efficiency (CCE) is defined as the ratio of the collected charges at the electrode $Q_{\text {coll }}$, and the generated charges $Q_{g e n}$ in the sample i.e., $C C E=Q_{\text {coll }} / Q_{\text {gen }}$. Typically, CCE is in the range of 0.1 to 0.6 in pcCVD as opposed to 1 for the single crystal CVD (scCVD) diamond. However, the poor CCE for the pcCVD diamond does not prevent it from achieving the time resolutions required for the proposed application, provided the charge deposited is sufficiently high. Furthermore, scCVD diamond is not available in large area samples and so, cannot be used for our purpose.

In this paper we present our recent study of the effect of electrode fabrication, and size (i.e. detector capacitance) on the timing characteristics for large area pcCVD synthetic diamonds, as well as the influence of threshold and signal amplitudes on their performance. The work we 
present here adds a further dimension to the published data by providing a systematic experimental study of the capacitance effect for a series of custom made samples, fabricated and tested in identical conditions.

It is also worth noting that a recent and comprehensive experimental and theoretical review by Ciobanu et al. [21] summarises the ToF measurements carried out using sc/pc CVD detectors of various thicknesses and areas (i.e. different capacitances) with beams ranging from protons to heavy ions. Most of the results presented involved processing of detector signals at distances of few $\mathrm{mm}$ to $\mathrm{cm}$, usually on the PCB on which the detector is mounted. In our work, we process the signals "conventionally" with GHz preamps located at a distance of approximately $1 \mathrm{~m}$ from the detector.

Our results show that a large area pcCVD diamond detection system can provide the required high timing resolution under these conditions, i.e. for LYCCA or other related particletracking systems in nuclear physics.

\section{Material and Methods}

The present work mainly uses pcCVD synthetic diamond wafers with dimensions of $20 \mathrm{~mm}$ x $20 \mathrm{~mm}$ x $0.3 \mathrm{~mm}$, which were obtained from Diamond Detectors Limited, UK (DDL). Prior to electrical contact fabrication, samples were thoroughly cleaned for durations of 10,5 and 5 min. using aqua regia, acetone, and isopropanol, respectively. As shown in Fig. 1 (left), the metal layer forming the electric contact was deposited directly onto the wafer using the setup at the University of Surrey described later - the metallisation henceforth is referred to as 'Type 1'. The Type 1 contacts were fabricated in a "sandwich" configuration; one side having Pattern 1 (see Fig. 1) of 4 strips with an area of $81 \mathrm{~mm}^{2}(18.0 \mathrm{~mm} \mathrm{x} 4.5 \mathrm{~mm})$ per strip and a gap of $\sim 275 \mu \mathrm{m}$ between strips. The other side of the wafer was a planar ground pad of $18 \mathrm{~mm}$ x $18 \mathrm{~mm}$. Two diamond samples were fabricated with $100 \mathrm{~nm}$ thick Au contacts using a shadow mask and a K575XD Turbo Sputter Coater to deposit the metal. The remaining two samples were prepared with $50 \mathrm{~nm} \mathrm{Al}$ contacts that were deposited using photolithography in a thermal evaporator (Edwards E306), at a working pressure of $10^{-6}$ Torr.

As shown in Fig. 1 (Right), devices with gold contacts were then installed onto a printed circuit board (PCB) using conductive and adhesive, fast drying, silver paint. Subsequently, the top 4-strip contacts were connected to the PCB signal lines using gold wires of $24 \mu \mathrm{m}$ in diameter. The PCB has $50 \Omega$ impedance-controlled tracks, to reduce reflections on the same time-scale as the sub-nanosecond rise time (i.e. the time it takes for the pulse to rise from $10 \%$ to $90 \%$ of its maximum height) of the pulses.

To investigate the timing resolution as a function of detector capacitance, we also produced signal pads A, B, C and D of Type 1 with four different areas of 90, 50, 12 and $6 \mathrm{~mm}^{2}$ (corresponding to capacitances of 14.6, 8.1, 2.0 and $1.0 \mathrm{pF}$ ) respectively on one wafer. These pads are shown as Pattern 2 in Fig. 1. The ground pad in this case is the same as that for Pattern 1. Sensors with a capacitance of $13.5 \mathrm{pF}$ that had been fabricated by DDL [20] were also used. These had a different metallisation that we refer to as 'Type 2'. As can be seen in Fig. 1, 'Type 2' metallisation also has an additional Diamond-Like Carbon [DLC] layer between the diamond wafer and the Au contact. For more details see Galbiati et al. [20].

Prior to the experiments with the beam, sensors were systematically tested for variations in the applied bias using an ${ }^{241} \mathrm{Am}$ alpha particle source and a charge sensitive pre-amplifier. This helped us to assess the detectors stability and determine an optimal bias for the tests. In the bias 
range of $\pm 500 \mathrm{~V}$, the leakage current from individual strips was found to be $\sim 10 \mathrm{pA}$, leading to a bulk resistivity of $\sim 1.4 \cdot 10^{15} \Omega \cdot \mathrm{cm}$.

\section{Experimental Setup}

A typical arrangement of two PCBs in transmission geometry is shown in Fig. 1 (Right). Each of the PCBs holds two diamond wafers. Tests were carried out using particle beams from cyclotron facilities at Birmingham, UK and the Cyclotron Institute at Texas A\&M University (TAMU), USA, to evaluate timing performances for different amounts of energy deposition. In both cases, the beam particles passed through the first detector and were either stopped in (Birmingham), or passed through (TAMU) the second, allowing for a diamond-diamond coincidence measurement using identical detectors. In order to conform with the specific aims of the tests performed, different beam properties and setups were used at the two facilities. A brief description is given in the following sub-sections.

\subsection{Setup for measurements with $16.3 \mathrm{MeV} / \mathrm{u}^{3} \mathrm{He}$}

The Birmingham MC40 Cyclotron facility provided a ${ }^{3} \mathrm{He}$ beam at energy of $16.3 \mathrm{MeV} / \mathrm{u}$ (i.e. $50 \mathrm{MeV}$ ), which was used for two separate tests. For the first test, two diamond detectors (Type 1, Pattern 1) were mounted in transmission geometry onto two PCBs and irradiated with a scattered beam inside (see below) a vacuum chamber. It should be noted that, for this test only, the signal lines were not impedance controlled. In the second test, two sets of diamond detector pairs, mounted in transmission geometry onto two impedance controlled PCBs, were again placed inside a vacuum chamber and irradiated with the beam scattered from a $\mathrm{Pb}$ target at $\sim 45$ deg (Type 1) and 75 deg (Type 2) as shown in Fig. 2 (top).

As the scattered beam passed through, approximately half of the beam energy was deposited in the front wafer whilst the remaining energy was deposited in the back wafer. The charge collected at the metal contacts can be estimated by, $Q_{\text {coll }}=1.6 .10^{-19} \cdot C C E \cdot \Delta E / e_{p h}$. Using $13 \mathrm{eV}$ for the energy required to create an electron-hole pair $\left(e_{p h}\right)$, and typical values of 0.35 and 30 $\mathrm{MeV}$ for the $C C E$ and $\triangle E$ (energy deposited in the detector by the beam) respectively, we obtain $0.13 \mathrm{pC}$ for $Q_{\text {coll }}$. The detector signals were transferred using a cable of $\sim 1 \mathrm{~m}$ length from the PCB via SMA vacuum feed-through to the DBA IV [12], which was placed outside the vacuum chamber, for pre-amplification. In an ideal situation, for a pulse width of $1 \mathrm{~ns}$ and $50 \Omega$ impedance-controlled high frequency line, we expect to see a $0.65 \mathrm{~V}$ signal at the output of the DBA IV with a gain of $\mathrm{L}=50 \mathrm{~dB}$. For the calculation, we use $A_{\text {out }}=10^{L(\text { in } d B) / 20} A_{\text {in }}$, where $A_{\text {out }}$ and $A_{\text {in }}$ are detector pulse amplitudes at the input and output of the DBA IV respectively. The outputs from the DBA IV typically had amplitudes of $0.2 \mathrm{~V}$. The pulses were acquired in coincidence mode using a Tektronix TDS7254B $2.5 \mathrm{GHz}$ oscilloscope.

For all the measurements described here, the signals were transported from the detector to the DBA IV by cables of type RG-316 and SMA vacuum feedthroughs. These cables were specially chosen because they are rated for low attenuation losses at high frequency, and transmit the diamond timing signals with subsecond rise times without significantly altering their characteristics. Cable lengths were kept as short as possible, typically between 0.5 to 1 meters, to minimize any alteration of the timing quality of the diamond signals.

Customised LabVIEW software code, developed in house, was used to carry out an off-line analysis of the data, which was collected by the $2.5 \mathrm{GHz}$ oscilloscope. A pulse shape analysis 
was also done using ROOT [22] based software, which not only served as a crosscheck, but also gave the possibility to analyse using complementary algorithms. This enabled a coincidence analysis, which included the ability to correlate timing performance with pulse height and threshold level.

In a simultaneous and complimentary measurement with the same detectors used in the second test, signals from the DBA IVs were processed through a dedicated setup of electronics and data-acquisition shown in Fig. 3. The current pulse produced within a diamond strip was transferred in an impedance-controlled manner to the DBA IV [12]. The HV input on the DBA IV allowed us to provide a voltage in the range of 300 to $1000 \mathrm{~V}$ to the diamond wafer. The DBA IV output was then fed to leading edge discriminators (LEDs), the thresholds on which could be set to as low as $-10 \mathrm{mV}$ with high stability. The NIM standard outputs from the LEDs were then converted to the ECL standard using a NIM-ECL converter, before getting fed to the time to digital converter (TDC) V1290A Caen module [23] in its high-resolution mode. The TDC can record times with a dispersion of 25 ps per channel and its trigger was set so as to collect the data only when at least one of the LED channels had beam related data (i.e., OR (LED)). As shown in Fig. 3, this was achieved using the SAC module [24] which distributes the trigger required by the data acquisition system in order to start the data collection. The OR (LED) was fed to the SAC module as the trigger, which in turn provides the Monitor (M2) signal that was shaped using the gate and delay generator (GDG) module. A coincidence signal of shaped M2 and the delayed OR (LED) was fed as a trigger to the TDC. The data was then transferred to our multi instance data acquisition system (MIDAS) [25] for storage and analysis. In this case only the timing information was recorded and for an off-line analysis using a custommade software code.

\subsection{Setup for measurements with ${ }^{40} \mathrm{Ar}$ and ${ }^{20}$ Ne beams}

The cyclotron institute located at TAMU provided suitable beams of different atomic number that were selected by the MARS separator [26]. The beam energies are higher compared to that for ${ }^{3} \mathrm{He}$ from Birmingham cyclotron, and the energy loss in the detectors was much closer to the anticipated energy deposition at fragmentation facilities such as the Super FRS at FAIR, simulating more realistic conditions expected for the LYCCA applications. Four diamond sensors of Type 1, Pattern 1 were again installed in pairs on two-layer $50 \Omega$ impedance controlled PCBs as shown in Fig. 1 (Right). Each of the front and back PCBs has one Au and one Al metallised wafer with a total of eight detector strips. The two-layer PCBs were then arranged in a transmission geometry separated by 14.5 (or 29.5) $\mathrm{mm}$ with a good alignment between the strips of same metallisation, Type 1 and were placed inside the vacuum chamber as shown in Fig. 2 (bottom). A secondary beam, mainly consisting of $35 \mathrm{MeV} / \mathrm{u}{ }^{40} \mathrm{Ar}$ ions, deposited approximately 500 and $750 \mathrm{MeV}$ of energy as the ions passed through the front and back detectors respectively, producing signals with amplitudes of $\sim 2 \mathrm{~V}$. The post-preamplifier noise levels were typically around 30-40 mV. The DBA IV pre-amplified the fast current signals and the same setup of electronics as described in section 3.1 was used, together with the MIDAS for data acquisition.

A $25 \mathrm{MeV} / \mathrm{u}{ }^{20} \mathrm{Ne}$ primary beam was also used at TAMU, which after impinging on the cryogenic $\mathrm{H} 2$ target, generated secondary beams with the same mass to charge ratio of $q / m=1 / 2$, but with three different magnetic rigidities. This resulted in secondary beams that were mixtures of ${ }^{20} \mathrm{Ne}$ (from inelastic scattering) and ${ }^{16} \mathrm{O}$ (from the break-up of the ${ }^{20} \mathrm{Ne}$ projectiles) at 3 
energies of 22.5, 21.5 and $20.8 \mathrm{MeV} / \mathrm{u}$, with different mixing ratios. Measurements were mainly made at $20.8 \mathrm{MeV} / \mathrm{u}$ where the two components were almost equal.

\section{Results and Discussion}

We investigated the timing properties of two diamond sensors metallised with Au contacts as a function of the applied bias voltage using a $16.3 \mathrm{MeV} / \mathrm{u}{ }^{3} \mathrm{He}$ beam. The strips on the front detector were kept at $+600 \mathrm{~V}$ whilst the bias of the strips on the back detector was varied from $+600 \mathrm{~V}$ to $+1000 \mathrm{~V}$. Signals typically with amplitudes of $0.1-0.2 \mathrm{~V}$ and a noise level of $20 \mathrm{mV}$ were observed at the output of the DBA IV. In the LabVIEW analysis, the baselines of these signals were adjusted and matched. The time at which the signal reaches $30 \mathrm{mV}$ (which is just above the $20 \mathrm{mV}$ noise) was obtained for each pulse. A histogram of the calculated times of flight was created from their differences, i.e. the time-difference (TD) spectrum. A Gaussian fit to the ToF peak gave the time resolution, $\sigma_{\mathrm{TD}}$, and its error.

Fig. 4 shows the time resolution, $\sigma_{\mathrm{TD}}$, calculated from the pulse shape analysis for a front/back strip pair as a function of the applied bias. Each curve from the Birmingham data using ${ }^{3} \mathrm{He} 16.3 \mathrm{MeV} / \mathrm{u}$ beam corresponds to a different signal/noise $(\mathrm{S} / \mathrm{N})$ ratio, which is proportional to the signal amplitude. A higher value for $\mathrm{S} / \mathrm{N}$ implies higher signal amplitude, since the variation in the noise levels was negligible. It should be noted that, for this case, a PCB was used without impedance controlled signal lines. Fig. 4 reveals that $\sigma_{\mathrm{TD}}$ a) does not show any clear trend with respect to the applied bias and stays nearly constant between $+600 \mathrm{~V}$ and +1000 $\mathrm{V}$ - possibly due to the saturation of the charge carrier velocities at high bias, $b$ ) is lower for higher $\mathrm{S} / \mathrm{N}$ values (or higher signal amplitudes) at a fixed bias, c) is $\sim 128$ ps per pair (or the time resolution per detector strip, $\sigma_{\mathrm{t}} \sim 128 \mathrm{ps} / \sqrt{ } 2=90 \mathrm{ps}$ ) for high signal amplitudes.

The resolution in the ToF spectra, $\sigma_{\mathrm{TD}}$, can be improved by minimizing the contributions from the 'walk' and 'jitter' components, which depend on the detector and pre-amplifier characteristics respectively. The contribution from electronics can be given as [21]:

$$
\sigma_{t e}=\delta t+\frac{\sigma_{n}}{d V / d t}=\delta t+\frac{\sqrt{K T / C_{D}}}{2.28 Q_{\text {coll }} B W / C_{D}}
$$

where $\sigma_{n}, d V / d t, K, T, Q_{\text {coll }}, B W$ and $C_{D}$ correspond to noise dispersion, the slope of the signal at the discriminator threshold, Boltzmann constant, temperature, the collected charge, band width of the pre-amplifier and the capacitance of the detector respectively. $\delta \mathrm{t}$ is the contribution from intrinsic jitter of discriminator and time digitizer. The formula assumes that no parasitic capacitances are present in the setup. Clearly, the timing resolution improves as the charge collected by the metal contacts increases, which has also been observed in Refs. [18,21]. We therefore expect an improved $\sigma_{\mathrm{TD}}$, for relativistic heavy ions, where the energy deposition in the sensors is considerably higher.

Fig. 5 (left) shows the pulse shapes from pads biased at $+400 \mathrm{~V}$ with different capacitances, $C_{D}$. As can be seen, the rise time increases with $C_{D}$. Our results presented in Fig. 5 (right) show that the timing resolution, $\sigma_{\mathrm{TD}}$, improves when the capacitance is reduced. Clearly, it is preferable to keep the capacitance as low as practically possible. This is in line with equation (1), from which it is expected that $\sigma_{\mathrm{TD}}$ is proportional to $C_{D}{ }^{1 / 2}$. We also consistently observed a better performance for Type 2 contacts and of all the detectors tested until now the Type 2 devices gave the best results. 
For the $35 \mathrm{MeV} / \mathrm{u}{ }^{40} \mathrm{Ar}$ measurements at TAMU, the energy losses in the sensor are greater than $500 \mathrm{MeV}$, which produce signals of $\sim 2 \mathrm{~V}$ amplitude with a noise level of $\sim 40 \mathrm{mV}$ at the output of DBA IV. The leading edge discriminator thresholds were therefore adjusted to be at 40 $\mathrm{mV}$. Some of the front strips were found to have poor performance, possibly due to the quality of the metal contact and signal lines, and therefore here we show the data from the best performing strips.

As can be seen in Fig. 4, the time resolution, $\sigma_{\mathrm{TD}}$, for a front/back Au strip pair from the ${ }^{40} \mathrm{Ar}$ data improves as a function of the applied bias between $+300 \mathrm{~V}$ to $+600 \mathrm{~V}$. Of the two data points at $+400 \mathrm{~V}$ (shown in Fig. 4 at +390 and $+410 \mathrm{~V}$ for clarity), a better timing resolution was seen when we had improved noise conditions emphasising the importance of low noise levels. Fig. 6 (right inset) shows an overall total timing resolution of $\sigma_{\mathrm{TD}}=62 \pm 5 \mathrm{ps}$, at $+470 \mathrm{~V}$ bias applied to each of the strips. Assuming equal contributions, a resolution of $\sigma_{t}=44 \pm 5$ ps was found for each detector strip. Such values are numerically comparable with those reported by Ciobanu et al. [21] although it should be noted that in our case, much higher energies were deposited in the sensors, and the detector signal processing was performed one meter away, i.e. not very close to the diamond detectors.

The same strip pair used with a slightly higher LED threshold level of $50 \mathrm{mV}$ gave somewhat worse value of timing resolution, $\sigma_{\mathrm{t}}=53 \pm 5 \mathrm{ps}$. This emphasizes the importance of low thresholds to minimize 'walk' effects. Consequently, we use LEDs that give the best possibility to use low thresholds.

For the cases of two Al strip pairs, we obtained $\sigma_{\mathrm{TD}}=102.0$ and $99.0 \mathrm{ps},\left(\sigma_{\mathrm{t}}=72.1\right.$ and 70.0 ps) respectively. No information was obtained from the other Al strip pairs due to their very low number of pulses detected. This could either be caused by the influences of the Al-diamond interface [20], or the oxidation of $\mathrm{Al}$ over time. It could also be related to the poor formation of the Al layer. Obviously, this needs confirmation by future experiments. From these results, we conclude that better timing resolutions were achieved using the data for diamond detectors metallised with Au contacts.

In order to investigate the timing characteristics at different signal amplitudes, we utilized the capability of the accelerator setup to provide a wide variety of beams and at different energies. A $20.8 \mathrm{MeV} / \mathrm{u}$ mixed beam of ${ }^{20} \mathrm{Ne}$ and ${ }^{16} \mathrm{O}$ from the cyclotron was also sent through the detectors. In this case the energy losses in the sensors (therefore the signal amplitudes) were lower compared to those for ${ }^{40} \mathrm{Ar}$ beam. For example ${ }^{20} \mathrm{Ne}$ deposited energies of 320 and 100 $\mathrm{MeV}$ in the front and back detectors, respectively. Even though the speed of both ${ }^{20} \mathrm{Ne}$ and ${ }^{16} \mathrm{O}$ is the same at the entrance of the first detector, the difference in energy loss through the detector means they travel with different speeds from the exit of the first detector to the entrance of the second detector. For the case of a flight path of only $14.5 \mathrm{~mm}$, this fact led to the separation of the two peaks by $\sim 150 \mathrm{ps}$ in the timing histogram, demonstrating the remarkable capability of the detectors to distinguish between ${ }^{20} \mathrm{Ne}$ and ${ }^{16} \mathrm{O}$. As shown in Fig. 6, a separation of $\sim 300 \mathrm{ps}$ between the corresponding peaks in the ToF spectrum was then achieved when the flight path was increased to $29.5 \mathrm{~mm}$. The resolutions, $\sigma_{\mathrm{TD}}$, for these two peaks are found to be around 81.0 and $96.0 \mathrm{ps}\left(\sigma_{\mathrm{t}}=57.3\right.$ and $\left.67.9 \mathrm{ps}\right)$.

We note that a component of the secondary ${ }^{39} \mathrm{Ar}$ beam was also present during our measurements with ${ }^{40} \mathrm{Ar}$ beam. We estimate over a flight path of $14.5 \mathrm{~mm}$, the two Ar species to have a separation in time of only $14 \mathrm{ps,} \mathrm{which} \mathrm{is} \mathrm{much} \mathrm{lower} \mathrm{than} \mathrm{the} \mathrm{resolution} \mathrm{achieved} \mathrm{in} \mathrm{the}$ current setup. Therefore, we do not expect to identify these two components using the ToF spectra. Such a contribution could cause a broadening of the timing resolution peak represented 
in Fig. 6 (inset). Therefore, in reality the diamond strips may have marginally better resolution than that obtained from the histogram.

\section{Conclusions}

In this paper, we presented preliminary results on time-of-flight measurements using large area polycrystalline CVD diamond detectors, manufactured with different types of metal contacts and capacitances. Diamond detectors were studied in relation to their timing resolution $\sigma_{\mathrm{t}}$ as a function of applied bias, capacitance and the energy deposited in the detectors.

Results, in agreement with those reported in literature for small area scCVD and pcCVD $[13,21]$, showed that low noise, low capacitances (i.e. smaller area of the electrodes) and high charge collection results in better timing performance.

Experiments performed with $16.3 \mathrm{MeV} / \mathrm{u}{ }^{3} \mathrm{He}$ beam showed that the timing resolution, $\sigma_{\mathrm{TD}}$, improves when the capacitance is reduced i.e. for diamond detectors having smaller size of electrodes. Moreover, the best timing performance was obtained with the devices provided by DDL with Type 2 contacts.

For energy losses of $\sim 600 \mathrm{MeV}$, from the $35 \mathrm{MeV} / \mathrm{u}{ }^{40} \mathrm{Ar}$ beam, diamond detectors fabricated with $\mathrm{Au}$ contacts gave better timing performance in comparison to those with $\mathrm{Al}$ contacts, achieving a timing resolution $\sigma_{t}$ of 44.0 ps. Consequently, ${ }^{20} \mathrm{Ne}$ and ${ }^{16} \mathrm{O}$ beam components could easily be distinguished over a flight path of only $\sim 14.5 \mathrm{~mm}$, and are well resolved for a path of $29.5 \mathrm{~mm}$. The study of timing performance of diamond detectors with different contact metallisation still requires further confirmation, and tests need to be repeated to optimize the devices.

Our data compare well with that presented in figure 22 of reference [21], which shows a strong dependence of the timing resolution with the $\mathrm{Z}$ of the ion - and hence in general with the energy deposited in the sensor. Whilst our numerical values compare well, it is important to note some key differences with our data. Firstly, our data were obtained at significantly lower energies, hence with higher energy deposition in the detector. This would be expected to yield a better timing resolution because walk effects are reduced. Countering that are the effects of our large detector capacitance (larger than that for the data presented in [21]) and the large cablelengths to the processing electronics, both of which might be expected to worsen the resolution, the latter of which will cause noise and degradation of the high-frequency signals. These conditions (energy, detector size and electronics) are much more representative of the typical nuclear physics applications likely to be encountered using devices like LYCCA at the SuperFRS at FAIR. The net result is that under these different conditions, our numerical values ( $\sigma_{\mathrm{t}}$ of about 45 ps for $\mathrm{Z}=20$, about 60 ps for $\mathrm{Z}=8$ and 90 ps for $Z=2$ ) compare well with the established data on in-beam diamond monitors.

\section{Acknowledgements}

We would like to thank the Diamond Detector Limited, UK (DDL) for providing us with the detectors fabricated with Type 2 contacts. The authors from UK universities and institutions would like to acknowledge the financial support from UK-STFC as a part of the UK-NUSTAR project.

\section{References}


[1]http://www.gsi.de/portrait/fair_e.html,

http://www.gsi.de/forschung/fair_experiments/NUSTAR/index_e.html.

[2] http://www.frib.msu.edu/.

[3] D. Rudolph et al, LYCCA - the Lund-York-Cologne-Calorimeter: Identification of reaction products in HISPEC_DESPEC@NuSTAR, Technical Report V1.2, (June 2009), http://www.nuclear.lu.se/fileadmin/nuclear/Grundlaeggande/lycca-TDR.pdf.

[4] M. J. Taylor, M. A. Bentley, D. Rudolph, C. Fahlander, P. Golubev, R. Hoischen, P. Reiter, J. Gerl and M. Gorska, "A New Simulation Package to Model Detector Systems with Fragmentation Reactions and Ion Separators”, Nucl. Instr. and Meth. A 606, 589-597, (2009).

[5] P. Golubev et al, to be published.

[6] http://www.gsi.de/forschung/fair_experiments/superfrs/index_e.html.

[7]. Hoischen, S. Pietri, D. Rudolph, W. Prokopowicz, H. Schaffner, S. Emde, P. Golubev, A. Wendt, N. Kurz, H.J. Wollersheim, J. Gerl, "Fast timing with plastic scintillators for in-beam heavy-ion spectroscopy" Nucl. Instr. and Meth. A 654, 354 (2011).

[8] M. Pomorski, E. Berdermann, A. Caragheorgheopol, M. Ciobanu, M. Kiš, A. Artemiyanov, C. Nebel, P. Moritz, "Development of single-crystal CVD-diamond detectors for spectroscopy and timing”, Phys. Stat. Sol (a) 203 (12), 3152-3160, (2006).

[9] A. Stolz, M. Behravan, M. Regmi and B. Golding, "Heteroepitaxial diamond detectors for heavy ion beam tracking”, Diam. Relat. Mat., 15, 807-810, (2006).

[10] R. Kalish, "Diamond as a unique high-tech electronic material: difficulties and prospects", J. Phys. D: Appl. Phys., 40, 6467-6478, (2007).

[11] D. R. Kania, M. I. Landstrass, M. A. Plano, L. S. Pan and S. Han, "Diamond radiation detectors”, Diam. Relat. Mat., 2, 1012-1019, (1993).

[12] P. Moritz, E. Berdermann, K. Blasche, H. Stelzer and B. Vosset, "Broadband electronics for CVD-diamond detectors”, Diam. Relat. Mat. 10, 1765-1769, (2001).

[13] E. Berdermann, M. Pomorski, W. de Boer, M. Ciobanu, S. Dunst, C. Grah, M. Kiš, W. Koenig, W. Lange, W. Lohmann, R. Lovrinčić, P. Moritz, J. Morse, S. Mueller, A. Pucci, M. Schreck, S. Rahman, M. Träger, "Diamond detectors for hadron physics research", Diam. Relat. Mat., 19, 358-367, (2010).

[14] The RD42 Collaboration, DRDC/P65, CERN/DRDC 94-21, 1994.

[15] C. Mer, M. Pomorski, P. Bergonzo, D. Tromson, M. Rebisz, T. Domenech, J. C. Vuillemin, F. Foulon, M. Nesladek, O. A. Williams, and R. B. Jackman, "An Insight into neutron detection from polycrystalline CVD diamond films”, Diam. Relat. Mat., 13, 791-795, (2004).

[16] E. Berdermann K. Blasche, P. Moritz, H. Stelzer, B. Voss, "The use of CVD-diamond for heavy-ion detection”, Diam. Relat. Mat., 10, 1770-1777, (2001).

[17] M. Pomorski, E. Berdermann, M. Ciobanu, A.Martemyianov, P.Moritz, M. Rebisz, and B. Marczewska, "Characterisation of single crystal CVD diamond particle detectors for hadron physics experiments”, Phys. Stat. Sol. (a) 202 (11), 2199-2205 (2005).

[18] M. Pomorski, PhD thesis (2008), Goethe Universität Frankfurt.

[19] J. Isberg, J. Hammersberg, E. Johansson, T. Wikström, D. J. Twitchen, A. J. Whitehead, S. E. Coe and G. A. Scarsbrook, "High carrier mobility in single-crystal plasma deposited diamond”, Science, 297, 1670-1672, (2002).

[20] A. Galbiati, S. Lynn, K. Oliver, F. Schirru, T. Nowak, B. Marczewska, J. A. Dueńas, R. Berjillos, I. Martel, and L. Lavergne, "Performance of Monocrystalline Diamond Radiation 
Detectors Fabricated Using TiW, Cr/Au and a Novel Ohmic DLC/Pt/Au Electrical Contact", IEEE TRANS. Nucl. Sci., 56, No. 4, 1863-1874, (2009).

[21] M. Ciobanu et al., IEEE Trans. Nucl. Sci., 54, No. 4 (2007), 58, No. 4 (2011).

[22] http://root.cern.ch.

[23] http://www.caen.it/servlet/checkCaenManualFile?Id=8082

[24] http://npg.dl.ac.uk/MIDAS/VME/sac.html.

[25] http://npg.dl.ac.uk/MIDAS/download/DataAcqApplications.html.

[26] R. E. Tribble, R. H. Burch and C. A. Gagliardi, "Mars: a momentum achromat recoil spectrometer”, Nucl. Instr. Meth A 285, 441-446, (1989).

\section{Figures}

Type 1
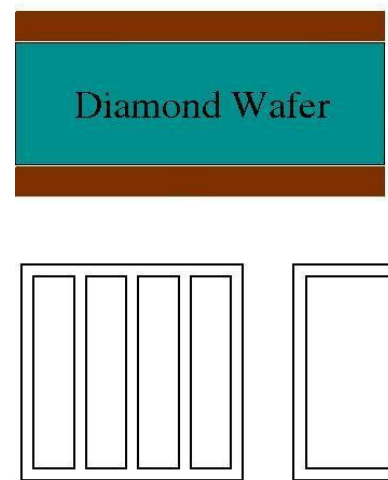

Pattern 1

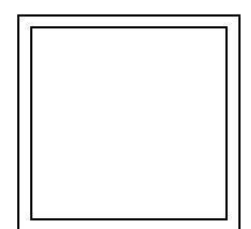

Ground Pad
Type 2
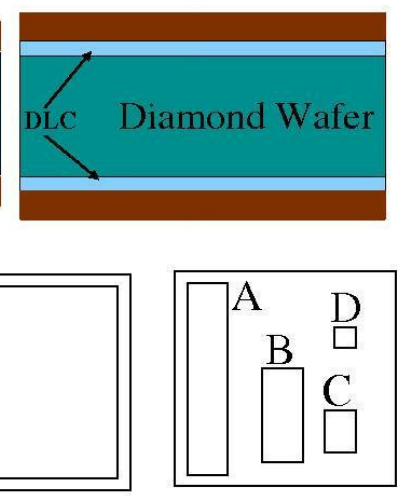

Pattern 2

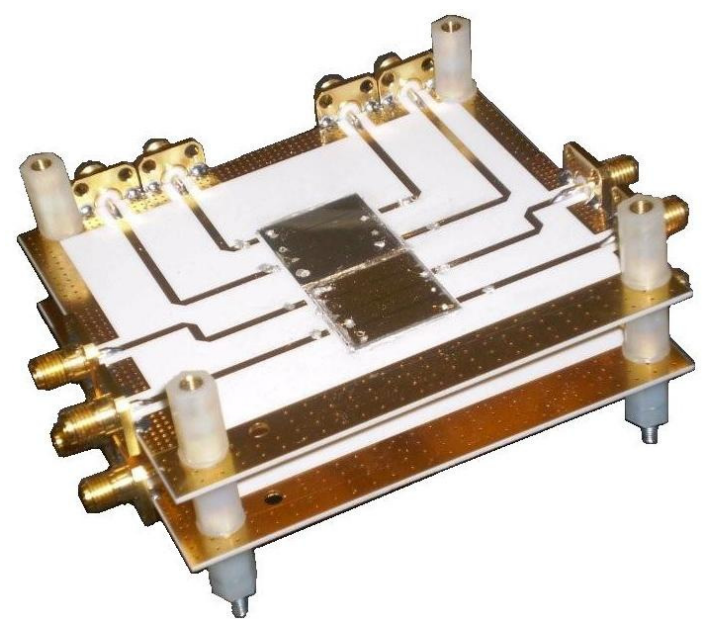

Fig. 1. (Left) On the top, schematic of the metal contacts Type 1 and Type 2 fabricated at University of Surrey and at Diamond Detector Limited respectively. Type 2 includes a Diamond Like Carbon [DLC] layer between the diamond wafer and the Au contact. At the bottom, Pattern 1 and Pattern 2 are shown together with the Ground Pad. See the text for more details. (Right) Diamond devices with $\mathrm{Al}$ and $\mathrm{Au}$ contacts mounted on two way printed circuit boards with adapted impedance of $50 \Omega$. The two PCBs separated by $\sim 14.5 \mathrm{~mm}$ in typical transmission geometry is shown. Signals are read out from the boards using high-frequency rated SMA connectors. 


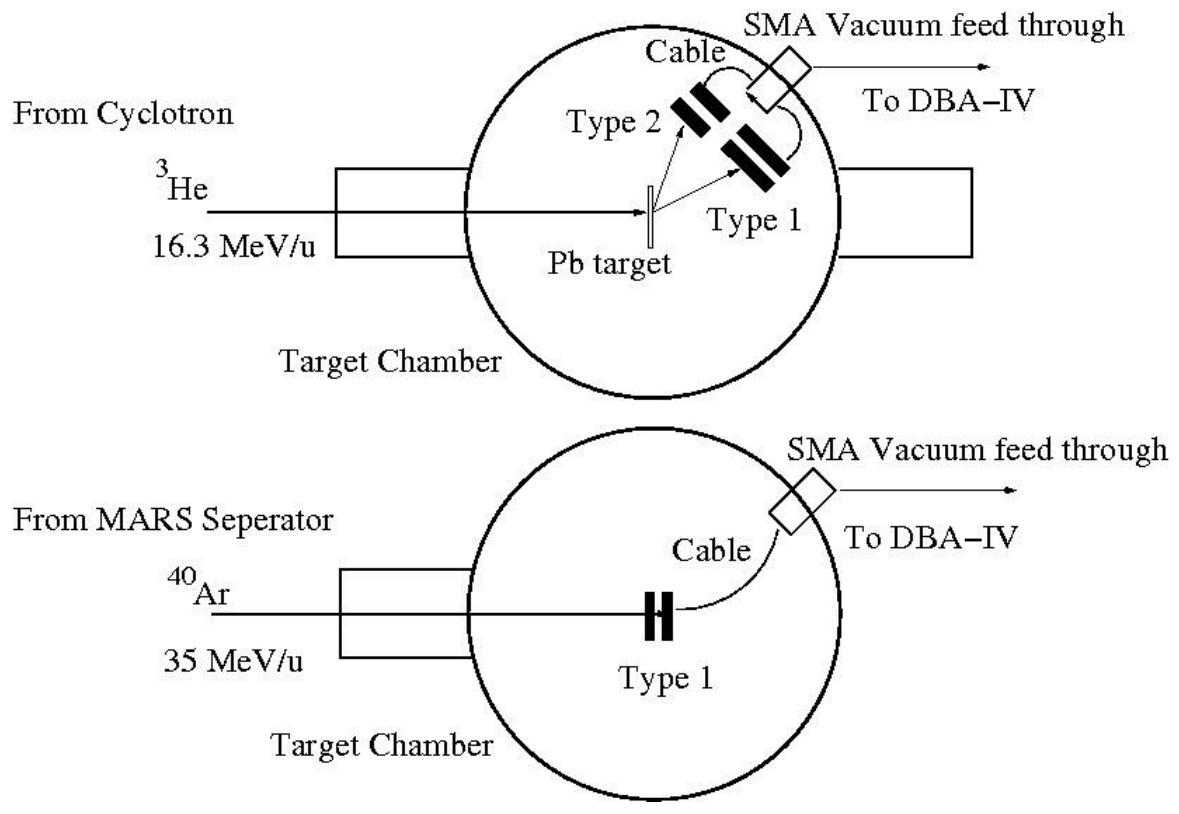

Fig. 2. (Top) The experimental setup used at Birmingham laboratory. (Bottom) The experimental setup used at TAMU facility. The target chambers are under vacuum. The detector signals are taken out of the vacuum via SMA feed-through to DBA IV preamplifiers. See the text for more details.

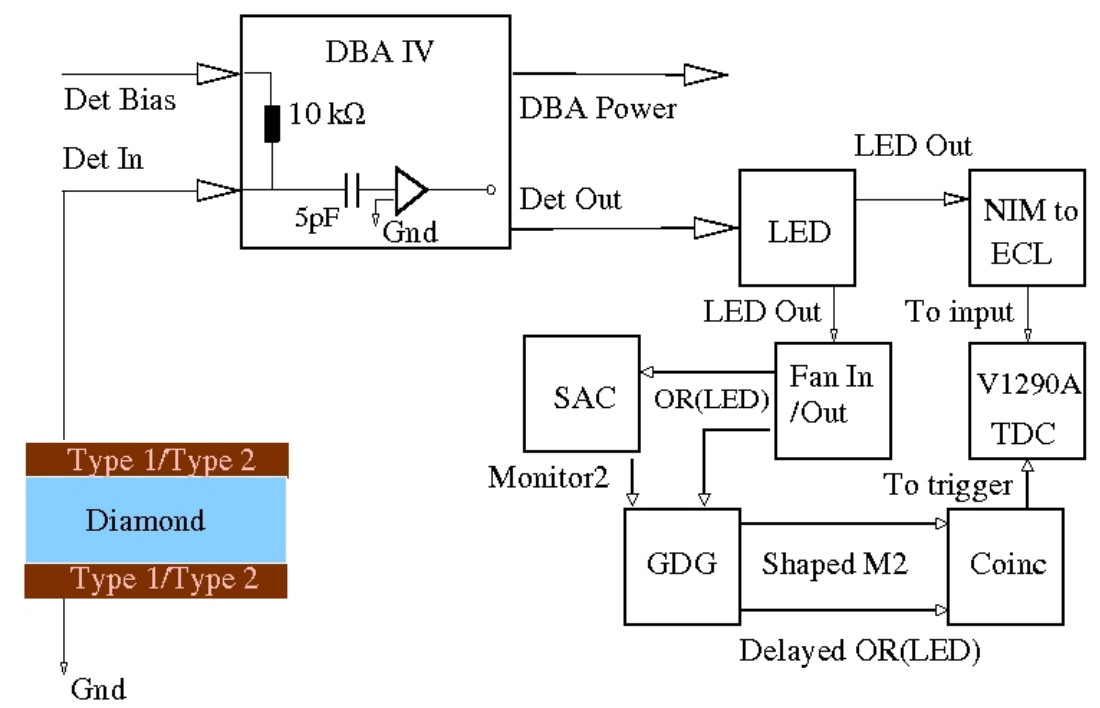

Fig. 3. Schematic of the electronic setup used for the post-processing of the DBA IV outputs. See the text for details. 


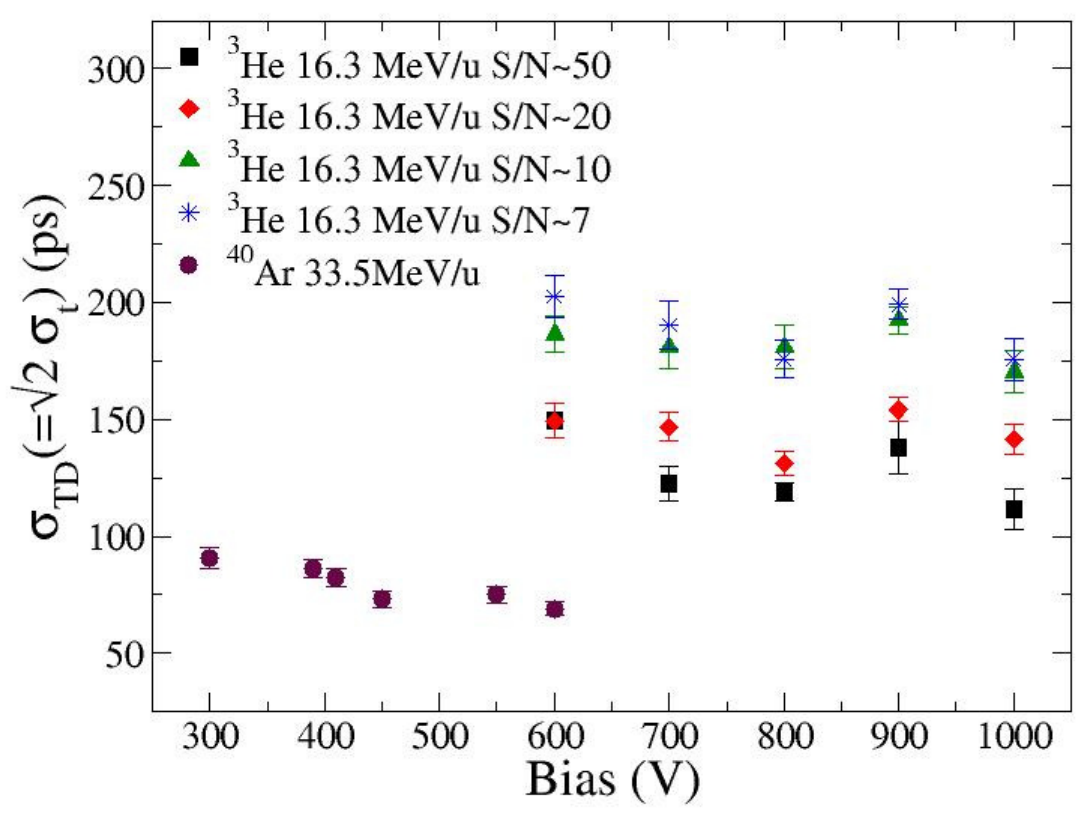

Fig. 4. Time resolution of a gold strip pair as a function of the applied bias at various signal/noise $(\mathrm{S} / \mathrm{N})$ ratio values. The ${ }^{3} \mathrm{He}$ data was taken with PCBs using signal lines that were not impedance controlled, and the bias was changed on the back detector strip between $+600 \mathrm{~V}$ and $+1000 \mathrm{~V}$ whilst keeping the front detector bias constant at $+600 \mathrm{~V}$. It should be noted that higher values of $\mathrm{S} / \mathrm{N}$ correspond to the selection of signals with higher amplitude. As can be seen, for 50 and 20 of $\mathrm{S} / \mathrm{N}$ values, $\sigma_{\mathrm{TD}} \sim 128$ ps can be achieved. The two data points shown at +390 and $+410 \mathrm{~V}$ for clarity were actually taken at $+400 \mathrm{~V}$. The better resolution achieved for the data point shown at $+410 \mathrm{~V}$ is due to improved noise conditions, which emphases the importance of achieving low noise levels in the setup. For ${ }^{40} \mathrm{Ar}$ data, impedance controlled PCBs were used and the timing resolution corresponds to a front/back strip pair with Au contacts, obtained using an electronics setup that used a TDC. Consequently, off-line analysis of the pulse shapes was not possible.
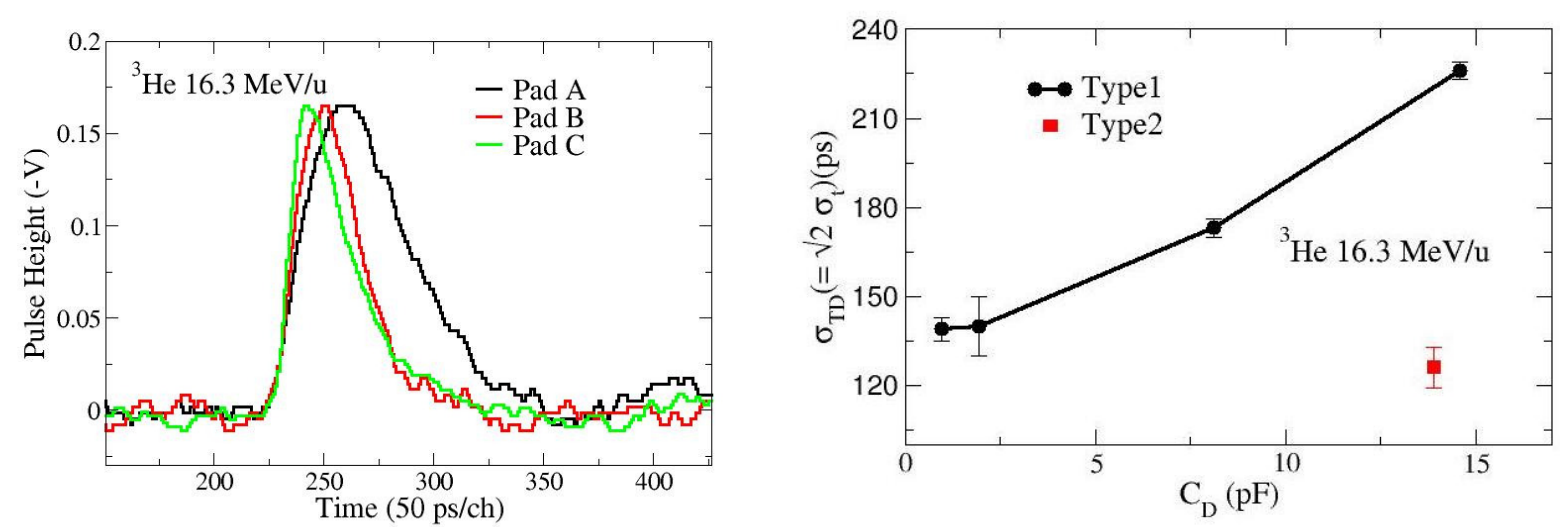
Fig. 5 (Left) Pulse shapes for the pads A, B and C. As can be observed, the rise time is faster for the pad with smaller area. (Right) Timing resolutions of diamond strips with different capacitances. As can be seen, lower capacitance gives better timing characteristics. It can also be noted that the Type 2 contact with a diamond-like carbon layer gives far better resolution.

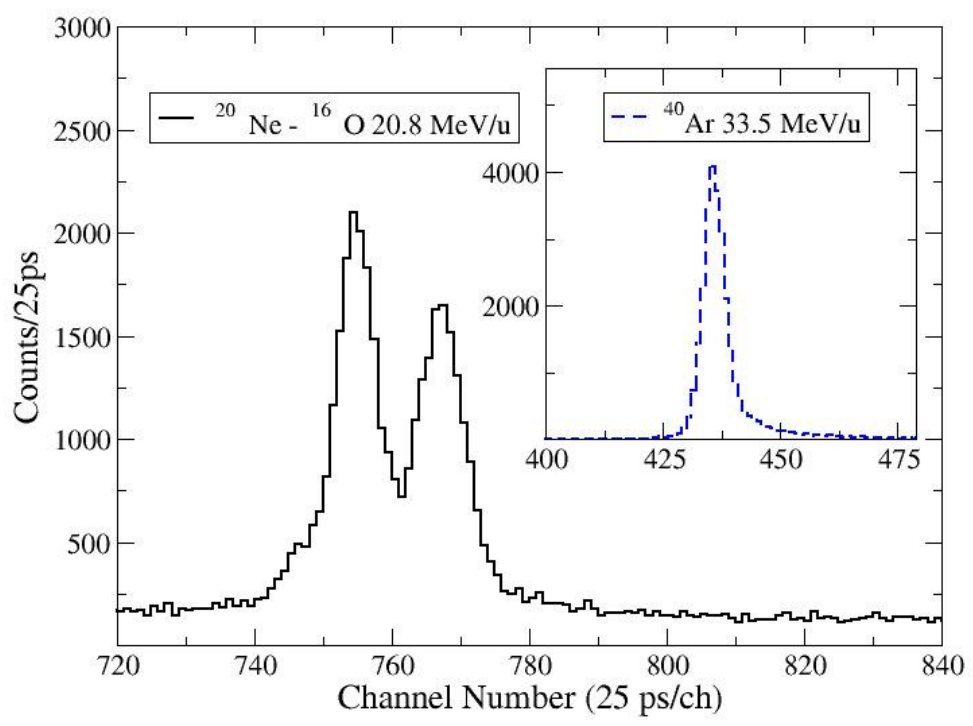

Fig. $6 .{ }^{20} \mathrm{Ne}$ and ${ }^{16} \mathrm{O}$ beam components revealed as two separate ToF peaks with $\sigma_{\mathrm{TD}}$ values of 81.0 and $96.0 \mathrm{ps}\left(\sigma_{\mathrm{t}}=57.3\right.$ and $\left.67.9 \mathrm{ps}\right)$ respectively when the diamond detector strip pair was separated by $29.5 \mathrm{~mm}$. A histogram for the case of $14.5 \mathrm{~mm}$ separation, where the two peaks could also be distinguished, is not shown here. Histogram in the inset shows the time difference between a gold front/back strip pair signals with a bias of $+470 \mathrm{~V}$ and LED threshold at $40 \mathrm{mV}$. It results in $\sigma_{\mathrm{TD}}=62$ ps per pair, implying $\sigma_{\mathrm{t}}=43.9$ ps per detector when the two strips are assumed to have identical properties. It should be noted that this is the best resolution obtained using the data from TAMU experiment. 